\title{
Correction to: Eribulin, trastuzumab, and pertuzumab as first-line therapy for patients with HER2-positive metastatic breast cancer: a phase II, multicenter, collaborative, open-label, single-arm clinical trial
}

\author{
Kenichi Inoue ${ }^{1}$ (D) Jun Ninomiya ${ }^{2} \cdot$ Tsuyoshi Saito $^{3} \cdot$ Katsuhiko Okubo $^{4} \cdot$ Takashi Nakakuma $^{5} \cdot$ Hirofumi Yamada $^{6}$. \\ Kei Kimizuka ${ }^{7}$. Tohru Higuchi ${ }^{3}$ - for the SBCCSG-36 investigators
}

Published online: 25 April 2019

(C) Springer Science+Business Media, LLC, part of Springer Nature 2019

Correction to: Investigational New Drugs

https://doi.org/10.1007/s10637-019-00755-x

The authors would like to note the replacement of Fig. 2b, for which Fig. 2a was placed erringly, with appropriate Fig. $2 b$.

The original article has been corrected.

The online version of the original article can be found at https://doi.org/ 10.1007/s10637-019-00755-x

Kenichi Inoue

ino.bad.ken@gmail.com

1 Division of Breast Oncology, Saitama Cancer Center, 780 Komuro, Ina-machi, Kita-adachi-gun, Saitama 362-0806, Japan

2 Department of Breast Surgery, Ninomiya Hospital, Soka, Japan

3 Department of Breast Surgery, Saitama Red Cross Hospital, Saitama, Japan

4 Department of Breast Unit, Toda Central General Hospital, Saitama, Japan

5 Department of Breast Surgery, Ageo Central General Hospital, Ageo, Japan

6 Department of Surgery, Sekishindo Hospital, Saitama, Japan

7 Department of Breast Surgery, Kasukabe Medical Center, Kasukabe, Japan 
Fig. 2 a Time to treatment failure with eribulin. b Time to treatment failure with trastuzumab and pertuzumab a

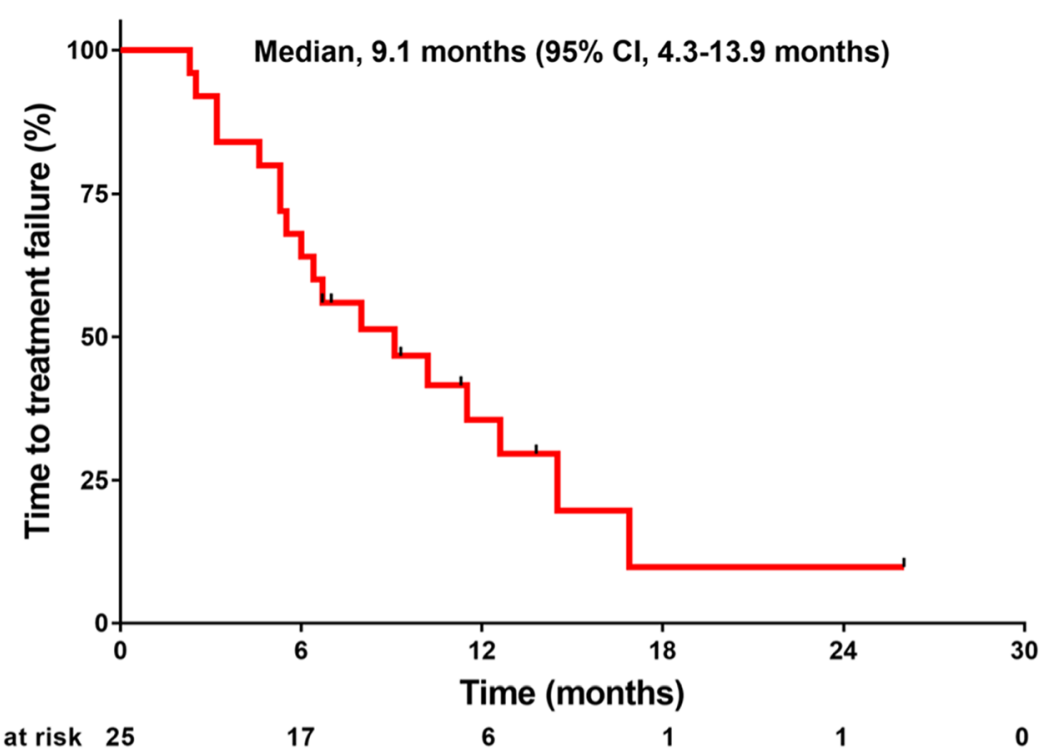

b

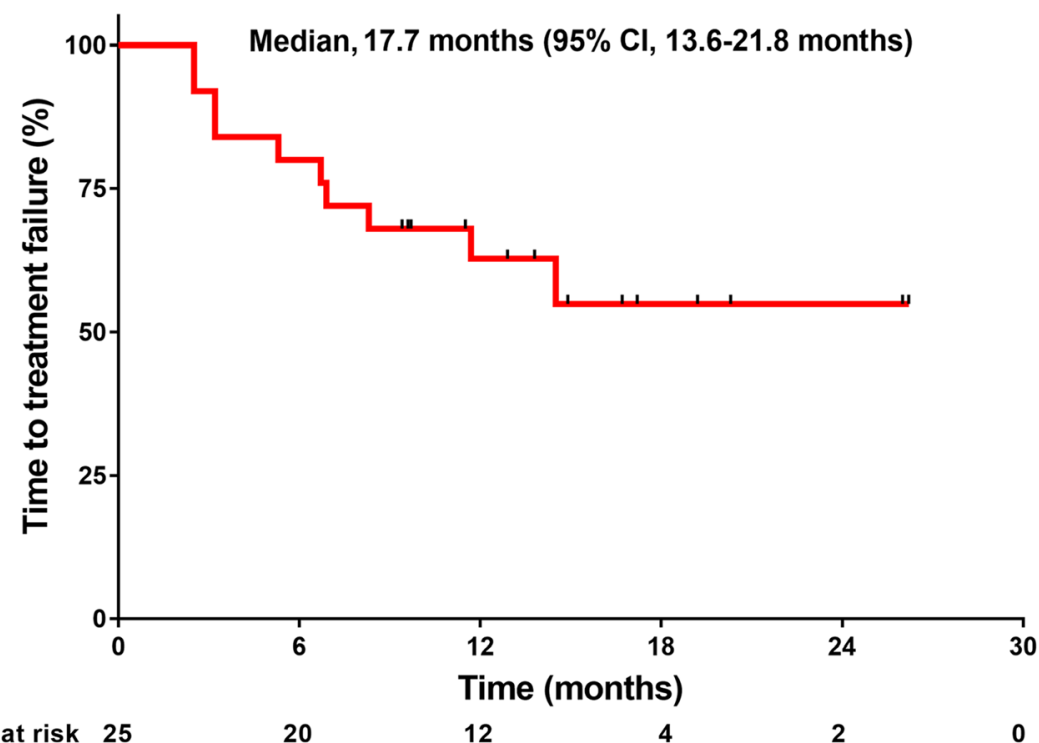

Publisher's note Springer Nature remains neutral with regard to jurisdictional claims in published maps and institutional affiliations. 